\title{
Prelmplantation factor (PIF) potentiates static magnetic field (SMF) effect to decrease tumor burden (melanoma murine model)
}

\author{
Beatrix Kotlan ${ }^{1,2^{*}}$, Janos F Laszlo ${ }^{3}$, Jozsef Tovari ${ }^{4}$, Miklos Kasler ${ }^{5,6}$, Eytan Barnea ${ }^{7,8}$ \\ From Society for Immunotherapy of Cancer 28th Annual Meeting \\ National Harbor, MD, USA. 8-10 November 2013
}

\section{Background and objectives}

PreImplantation factor (PIF) secreted by viable embryos exerts essential regulatory role on global systemic immune response. Synthetic PIF (PIF) translates endogenous effects to immune disorder models. Metastatic melanoma displays tumor-immunological behaviour. Static magnetic field (SMF) affects inflammatory reactions. There is increased interest toward SMF's potential antitumor effects. Herein examined a novel anti-melanoma strategy using combined physical and immune-based therapy.

\section{Methods}

Daily whole-body SMF exposure, combined with subcutaneous PIF administration was examined in engrafted HT199 melanoma cells' progression, transplanted into NSG mice. PIF effect on unique tumor-associated antigen expression relevant for tumor proliferation/ invasion was examined in vitro using specific antibodies. Direct PIF anti-proliferative effects on several cancer cell lines were tested using MTT.

\section{Results}

PIF potentiates SMF beneficial effect by reducing tumor volume vs. control $(\mathrm{Mmax}=96 \%)$ on day 34. Metastatic spleen mass is reduced by SMF alone $(\mathrm{M}=59 \%)$ or combined with PIF $(\mathrm{M}=62 \%)$. Daily SMF exposure alone inhibits tumor outgrowth $(\mathrm{Mmax}=60 \%, \mathrm{~F} 5.32(\mathrm{P}<0.002)$ $=21.16)$ while in combination with PIF, effect is considerably potentiated $(\mathrm{M}=80 \%)$, F5.32 $(\mathrm{P}<0.0004)=34.84)$. PIF did not impair tumor antigen expression nor reduced significantly cultured tumor cell lines' proliferation.

\section{Conclusions}

Collectively, results indicate that PIF's potentiating antitumoral effect is mainly immune-regulatory, synergizing with SMF's pro-tumor necrosis properties. The preserved tumor-associated antigen expression is important for the maintained antitumor immune activity. Overall, combined physico / immune regulatory treatment represents a useful, promising novel avenue for anti-cancer strategy.

\section{Acknowledgments \\ Harry J Loyd Charitable Trust Melanoma Research Award, INNO 08-3-2009- 0248, Biolncept LLC - PIF proprietary.}

\section{Authors' details}

${ }^{1}$ Molecular Immunology and Toxicology, National Institute of Oncology, Budapest, Hungary. ${ }^{2}$ Surgical and Molecular Tumorpathology, National Institute of Oncology, Budapest, Hungary. ${ }^{3}$ Applied Mathematics and Probability Theory, University of Debrecen, Faculty of Informatics, Debrecen, Hungary. ${ }^{4}$ Experimental Pharmacology, National Institute of Oncology, Budapest, Hungary. ${ }^{5}$ Board of Directors, National Institute of Oncology, Budapest, Hungary. ${ }^{6}$ Univ Medicine and Pharmacy, Tirgu Mures, Romania. ${ }^{7}$ Society for the Investigation of Early Pregnancy, Cherry Hill, NJ, USA.

${ }^{8}$ Biolncept LLC, Cherry Hill, NJ, USA.

Published: 7 November 2013

\section{doi:10.1186/2051-1426-1-S1-P80}

Cite this article as: Kotlan et al.: Prelmplantation factor (PIF) potentiates static magnetic field (SMF) effect to decrease tumor burden (melanoma murine model). Journal for ImmunoTherapy of Cancer 2013 1(Suppl 1):P80.

'Molecular Immunology and Toxicology, National Institute of Oncology,

Budapest, Hungary

Full list of author information is available at the end of the article

(0) 2013 Kotlan et al; licensee BioMed Central Ltd. This is an Open Access article distributed under the terms of the Creative Commons Attribution License (http://creativecommons.org/licenses/by/2.0), which permits unrestricted use, distribution, and reproduction in any medium, provided the original work is properly cited. 